\title{
002 PP TOWARDS AN ANTHROPOLOGY OF CARE: CANCER CARE PRACTICES IN THE EVERYDAY
}

MI Arteaga Pérez. University College London, UK

\subsection{6/bmjopen-2015-UCLSymposiumAbstracts. 13}

A patient affected by cancer is never alone with his/her disease; his/her social surroundings shape and are shaped by the daily practices aiming to manage the disease and its treatments. According to Macmillan Cancer Support report ${ }^{a}$, informal caregivers of colorectal cancer patients tend to provide more hours per week of care than those supporting patients affected by other types of cancer ( $40 \%$ of them give at least 20 hours of care versus $25 \%$ of all carers). Such care usually requires performing multiple tasks, from emotional support, practical work to advocacy.

Psychosocial studies in oncology have successfully attended to the repercussions of cancer and cancer care in the family unit, emphasising the impact of such experiences and transactions for the mental and physical health of family members. In this abstract, I would like to contribute to that research by explaining how an anthropology of care can shed further light on these dynamics, unfolding the practical, cognitive and emotional aspects of care in the everyday contexts of people affected by cancer.

My argument is that an anthropology of care would illuminate: (1) the generation of embodied knowledge, emotion work and practical tinkering of caregiving practices by which requirements, side effects and consequences of colorectal cancer treatments are addressed, (2) the ways in which patients and their significant others negotiate the temporalities of treatments and the collective identities as supporters and patients during the day to day life, and (3) the ways in which social-cultural and political and economic arrangements shape people's experiences of cancer treatments.

aMore than a million: Understanding the UK's carers of people with cancer-a report by Ipsos Mori for Macmillan Cancer Support. 